\title{
1 Ein Fallbeispiel
}

\section{Fallvignette Peter}

Im September 2007 äußerte ein 15\%1/2-jähriger Junge - nennen wir inn Peter - im Rahmen eines Mediensuchtprojektes seine Befürchtung, Amoktäter zu werden.

In einer sich anschließenden zweijährigen ambulanten Behandlung konnten Genese und Dynamik seiner Emotionen, Gedanken und Handlungsweisen besser verstanden und ein Einblick in die neue und spezifische Klientelgruppe der Schul-Amokläufer und School Shooter gewonnen werden.

In Peters imaginärem „Opferpool“ befanden sich einige Lehrer, von denen er sich gedemütigt fühlte, sowie Klassenkameraden, von denen er sich gemobbt sah, und ein für ihn „unerreichbares “ Mädchen. In Situationen, in denen er sich verzweifelt, depressiv und ausgeschlossen fühlte, beschäftigte er sich mit Gedanken an eine Amoktat. Dabei steigerte er sich mit „Ballerspielen“, aber auch mit Hilfe von Alkohol und Drogen in einen Aggressionsrausch. Dieses Verhalten wurde ihm phasenweise zu einem wichtigen Ventil für seine Aggressionen.

Nach dem Schul-Amoklauf von Winnenden (2009), so berichtete er, habe er stundenlang ein „komisches Gefühl, ein Kribbeln, in seinem Körper“ gespürt. Er habe den Täter Tim K. für seine Tat bewundert, aber auch einen „Heulkrampf“ über die Erkenntnis bekommen, „wie krank er selbst sei“.

Im Verlauf der Behandlung stellte sich heraus, dass Peters „Belastungsrucksack“ (Abb. 1) nahezu alle Aspekte enthielt, die in der Ursachenforschung zum Thema „Amok, School Shooting und zielgerichtete Gewalt“ allgemein diskutiert werden. Es schätzte den subjektiv empfundenen „Druck“ der einzelnen Belastungsteile nach der SUD(Subjective Units of Distress)-Skala (SUD 0 - SUD 10) ein. 


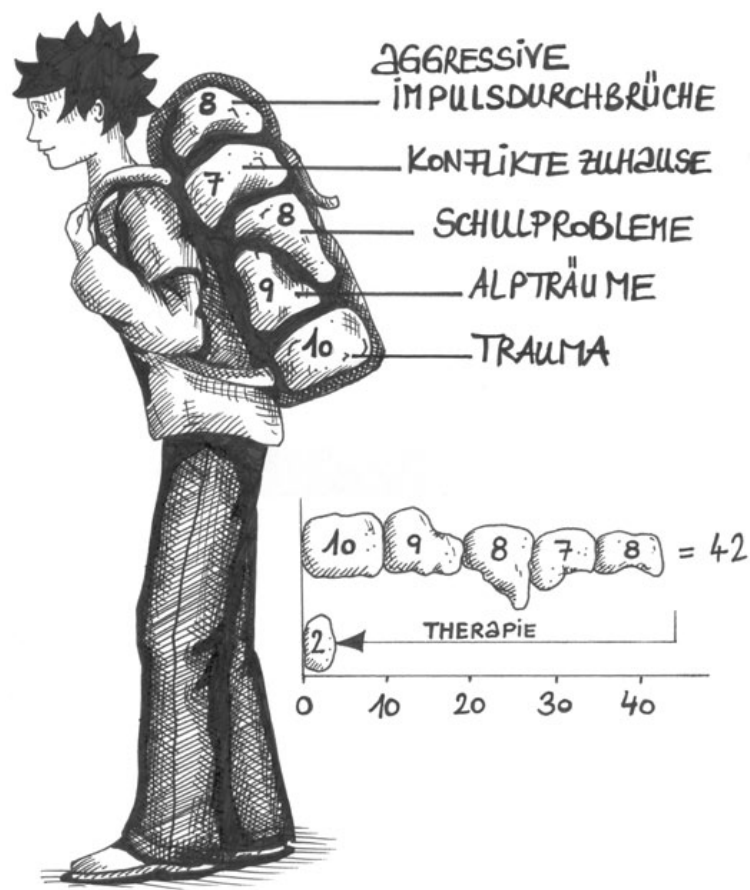

Abb. 1 „Belastungsrucksack“ mit SUD-Skalierung

\section{Inhalte von Peters „Belastungsrucksack“}

- Angst vor der Schule; kein „Bock“ auf Schule (SUD 7)

- Computersucht (SUD 9)

- keine Unterstützung durch Lehrer (SUD 3)

- aggressive Impulsdurchbrüche, schon bei Kleinigkeiten (SUD 9)

- Konflikte mit Schwester (SUD 1)

- Konflikte mit Eltern (SUD 5)

- keine Freundin und das Gefühl abgelehnt zu werden (SUD 6)

- Erleiden körperlicher Gewalt und Erniedrigung in der Grundschule; über $1 \frac{1}{2}$ lahre lang (SUD 6)

- Ausschluss aus der aktuellen Klassengemeinschaft (SUD 7)

In therapeutischen Gesprächen, zu denen auch seine Eltern hinzugezogen wurden, konnte Peter seine stark belastete Beziehung zum Vater verbessern. Sein Selbstwertgefühl und die Kontrolle über seine aggressiven Impulse stabilisierten sich deutlich. Im Verlauf der Behandlung wurde zu seinem wichtigsten Kernsatz:

„Ich will lernen, mich zu akzeptieren, so wie ich bin. Ich will lernen, meine Stärken zu entdecken und weiterzuentwickeln“. 
Gegen Ende der Behandlung konnte Peter seine Gewaltfantasien, die er in einer Art „Gewaltfilm“ auslebte, mit Hilfe eines „imaginären Videos“ rückwärts laufen lassen, sie in eine neue „Kassette“ legen und als „Regisseur“ einen völlig neuen, positiven und zukunftsorientierten Film erstellen.

Während Peter „nur“ seine Affinität zu vorausgegangenen Schul-Amokläufen empfand und entsprechend therapiert werden konnte, fanden andere potenzielle Täter offenbar keine sachkundigen Ansprechpartner, die ihnen hätten helfen können, ihre Probleme zu bewältigen. Wie wäre es sonst dazu gekommen, dass allein in Deutschland in den Jahren 2002-2009 neun Schul-Amokläufe stattfanden und dabei über 100 Personen getötet wurden. Es muss also ein wichtiges Ziel sein, spezifische Zeichen und Hinweise, die erfahrungsgemäß von potenziellen Schul-Amokläufern ausgehen, frühzeitig zu erkennen und zu beachten.

\subsection{Kriminalstatistiken}

Nach Hoffmann et al. (2009) zeigt die Kriminalstatistik vor 1999 weltweit nur acht Fälle zielgerichteter Gewalt an Schulen, nach 1999-2009 dagegen 29 Fälle. Deutsche Schul-Amokläufer und School Shooter sind zu $57 \%$ Einzelgänger. Mit der Polizei kamen von ihnen 42,9\% in Konflikt. Etwa 50\% waren noch sexuell abstinent. 57,1\% äußerten im Vorfeld suizidale Gedanken. Während die Zahlen allgemeiner schwerer Gewalttaten von Jugendlichen in den vergangenen Jahren in Deutschland rückläufig sind, steigt ihre Zahl an Schulen seit etwa 10 Jahren an.

Über 100 mehr oder weniger blutige School Shootings sind weltweit bekannt (Langman 2009). 1999 erlangte die Columbine-High School in Colorado traurige Berühmtheit als „Mutter“ oder „Blaupause“ aller Schulmassaker. Dort erschossen die beiden Jugendlichen Eric Harris und Dylan Klebold zwölf Mitschüler sowie einen Lehrer und verwundeten 23 weitere Menschen, bevor sie ihrem eigenen Leben ein Ende setzten.

Das bislang größte Massaker verübte Cho S. im April 2007 auf dem Uni-Campus in Blacksburg (Virginia). Der Schulanschlag in St. Augustin bei Bonn, den die 16-jährigeTanja O. mittels Molotow-Cocktails und einer Gaspistole verüben wollte, konnte noch rechtzeitig verhindert werden. Tanja O. äußerte in einem Abschiedsbrief: „Ich will erst meine Mitschüler weinen sehen, dann scheide ich aus dem Leben".

Die Schulleitung war schon einige Tage vor der geplanten Tat von Schülern auf das auffällige Verhalten der 16-Jährigen hingewiesen worden, doch eine „Gefährderansprache“ sollte erst drei Tage später stattfinden, weil angeblich eine Fremdgefährdung nicht vorgelegen habe, sondern nur eine Selbstgefährdung. Ein Trugschluss, der fatale Folgen hätte haben können. 
Damals sagte die Schulministerin von NRW, Frau Sommer, dass man das Täterprofil erweitern müsse, da man „Mädchen bisher ausgeklammert“ habe. Statistisch gesehen waren bisher nur 4\% Mädchen an Schul-Amok und School Shootings beteiligt.

\subsection{Ansätze zum Verständnis der Gewaltdynamik von Schul-Amokläufern}

In erster Annäherung stellt man fest, dass nur ein Teil der Schul-Amokläufer in Familien des,,sozialen Brennpunkts“ aufwuchsen, schon frühe körperliche Gewalt erfuhren und bald selbst in der Schule durch delinquentes und dissoziales Verhalten auffielen. Die meisten Täter wuchsen in „intakten Familien“ auf, eckten in ihrem sozialen Umfeld nur selten an und blieben in der Schule meist unauffällig. Von außen gesehen lebten sie in einer „heilen Welt“, die sie aber schließlich - für ihre Umgebung unverständlich - durch einen Gewaltakt zerstörten.

Die praktische Erfahrung und die theoretische Beschäftigung mit dem Phänomen „Schul-Amok, School Shooting und zielgerichtete Gewalt" machen deutlich, dass ein Verständnis der spezifischen Dynamik dieser Gewaltakte wegen der Vielfältigkeit der Ursachen nur mit Hilfe eines interdisziplinären Ansatzes gelingen kann. Das gilt insbesondere wenn es um Schlussfolgerungen für die Behandlung von potenziellen Schul-Amokläufern und um die Verhinderung möglicher Gewalttaten dieser Art geht. Dabei irritiert jedoch die Feststellung, dass Schul-Amokläufer nicht dem typischen Bild des jugendlichen „kriminellen“ Gewalttäters entsprechen, wie man ihn als medizinischer Sachverständiger kennt. Offensichtlich haben wir es hier mit einem Typus jugendlicher Gewalttäter zu tun, deren Täterprofil unter ganz neuen Gesichtspunkten zu ermitteln ist Als Folge subjektiv empfundener seelischer Verletzungen empfinden sich diese Täter als „Rächer“ und als „Richter über Leben und Tod“. Während ihres Amoklaufs sind für sie alle Regeln und Maßstäbe zivilisierten Zusammenlebens außer Kraft gesetzt. Sie befinden sich in einem Ausnahmezustand des Bewusstseins, in dem ihnen jede Form von Empathie fehlt. Ihr Verhältnis zu den übrigen Menschen schrumpft zu einer „Täter-Opfer-Beziehung“. Dabei geraten sie in einen „Aktionsrausch“, in dem sie auch Personen umbringen, die nicht zum ursprünglichen „Opferbestand“ gehören. Sie fühlen sich „grandios“ und „allmächtig“, bevor sie sich am Ende meist selbst töten, um sich ein unübersehbares „Denkmal“ zu setzen.

Die Ursache von Schul-Amokläufen ist - so die Ergebnisse der aktuellen wissenschaftlichen Forschung - niemals monokausal. Ein Schul-Amoklauf ist nicht das Resultat einer plötzlichen Überforderung, die in einen eruptiven Ausbruch blinder Gewalt mündet. Bei den bekannt gewordenen Schul-Amokläufen handelte sich vielmehr um eine über einen längeren Zeitraum geplan- 
te Tat, der stets eine längere Entwicklungsphase vorausging, in der die potenziellen Täter empfanden, durch Mitschülerinnen und Mitschülern, durch Lehrpersonen und andere als Autoritäten erlebten Personen gedemütigt, gemobbt oder seelisch und körperlich verletzt worden zu sein. Bei der Beurteilung dieses Tätertyps kommt es also zur Betrachtung eines komplizierten und die Entwicklung eines heranwachsenden störenden sozialen Beziehungsgeflechts.

\section{Nicht der Schul-Amoktäter allein, auch sein soziales Umfeld soll in den Blickpunkt gelangen.}

\subsection{Schnelle Schuldzuweisungen}

Nach einem Schul-Amoklauf werden von der Öffentlichkeit die vermeintlichen Auslöser meist schnell „gefunden“. Mangelnde Erziehungsfähigkeiten der Eltern, Auswirkungen ungünstiger sozialer Verhältnisse, Umgang mit Gewaltspielen am PC oder eine grundsätzliche psychische Andersartigkeit der jugendlichen Täter werden in Rechnung gestellt. Bei genauerem Hinsehen macht man aber die Erfahrung, dass die „Wahrheit unter der Oberfläche“ wesentlich differenzierter ist. Ins Zentrum der Betrachtungen gerät mehr und mehr das Bild eines schon über längere Zeit leidenden Jugendlichen, der eigentlich Hilfe gebraucht hätte, aber nicht in der Lage war, sie einzufordern oder effektiv zu nutzen, obwohl Hilfsangebote zur Verfügung standen. So können auch potenzielle jugendliche Täter, die einer Behandlung zugeführt werden, meist kein Vertrauen zu ihrem Therapeuten aufbauen und diese keinen Zugang zu ihm finden. 